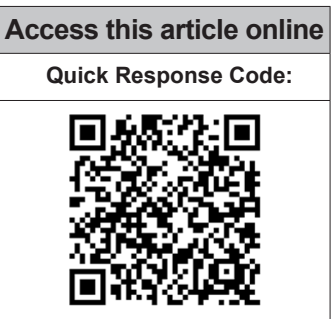

Website:

www.jponline.org

DOI:

10.4103/JLP.JLP_136_18
Department of Pharmacology, Toxicology and Medicine, College of Medicine Almustansiriya University, ${ }^{1}$ Department of Clinical Pharmacology, College of Medicine, Al-Mustansiriya University,

Baghdad, Iraq

Address for correspondence: Dr. Hayder M. Al-Kuraishy,

Department of Pharmacology, Toxicology and Medicine, College of Medicine Almustansiriya University, P.O.

Box 14132, Baghdad, Iraq. E-mail: hayderm36@ yahoo.com

Submission: 13-10-2018 Accepted: 25-06-2019

\title{
Renoprotective effect of irbesartan in a rat model of gentamicin-induced nephrotoxicity: Role of oxidative stress
}

\author{
Hayder M. Al-Kuraishy, Ali I. Al-Gareeb, Marwa S. Al-Naimi'
}

\begin{abstract}
:
BACKGROUND: The renin-angiotensin system (RAS) is essential in renal physiology; however, disturbance of the RAS is one of the chief pathways involved in renal injury. Dysregulation of RAS may result in both glomerular and tubulointerstitial injuries through direct effects of angiotensin II (Ang II) type 1 receptor. Irbesartan and other Ang II blockers have renoprotective effect through reduction of on renal inflammations. Therefore, the aim of the present study was to demonstrate the renoprotective effect of irbesartan on gentamicin-induced nephrotoxicity in rats concerning the oxidative stress.
\end{abstract}

MATERIALS AND METHODS: Thirty Sprague-Dawley Male rats divided into three groups, Group I (10 rats) treated with distilled water, Group II (10 rats) treated with gentamicin, and Group III (10 rats) treated with gentamicin plus irbesartan for 12 days. Blood urea, serum creatinine, serum malondialdehyde (MDA), superoxide dismutase (SOD), glutathione reductase (GSH), neutrophil gelatinase-associated lipocalin (NGAL), kidney injury molecules (KIM-1), and cystatin-c were measured in each group.

RESULTS: Irbesartan significantly reduced blood urea, serum creatinine, serum MDA, NGAL, KIM-1, and cystatin-c $P<0.05$. Irbesartan significantly increases SOD $P<0.05$ without significant effect in elevation of GSH serum levels.

CONCLUSION: Irbesartan has renoprotective effect in attenuation of acute nephrotoxicity through modulation of oxidative stress and antioxidant capacity in rats.

Key words:

Anti-oxidant, irbesartan, nephrotoxicities, oxidative stresses

\section{Introduction}

Nepri ephrotoxicity is a renal-specific circumstance in which excretions of toxic metabolites are considerably reduced due to toxic agents and drugs. ${ }^{[1]}$ Gentamicin is an aminoglycoside antibiotic that is very effective in treating life-threatening Gram-negative infections. Regrettably, $30 \%$ of patients treated with gentamicin for more than 1 week show some signs of nephrotoxicity. ${ }^{[2]}$ Gentamicin-induced nephrotoxicity (GIN) is characterized by direct tubular necrosis, mainly in the proximal tubule. The specificity

This is an open access journal, and articles are distributed under the terms of the Creative Commons Attribution-NonCommercial-ShareAlike 4.0 License, which allows others to remix, tweak, and build upon the work non-commercially, as long as appropriate credit is given and the new creations are licensed under the identical terms.

For reprints contact: reprints@medknow.com of gentamicin for renal toxicity is apparently related to its preferential accumulation in the renal proximal convoluted tubules. The exact mechanism of GIN is unknown. It has been reported that free radical formations are the major mechanism of GIN.${ }^{[3]}$ Certainly, gentamicin induces expression of cationic transporter proteins, namely megalin and cubilin at proximal renal tubules, which augment accumulation of gentamicin and free radical generations. In addition, gentamicin enhances the generation of reactive oxygen species causing deficiency in intrinsic antioxidant enzymes. In view of that, the use of some agents with antioxidant activity has been successfully used to ameliorate GIN. ${ }^{[4]}$

How to cite this article: Al-Kuraishy HM, Al-Gareeb Al, Al-Naimi MS. Renoprotective effect of irbesartan in a rat model of gentamicin-induced nephrotoxicity: Role of oxidative stress. J Lab Physicians 2019;11:200-5. 
The renin-angiotensin system (RAS) is essential in renal physiology; however, disturbance of the RAS is one of the chief pathways involved in renal injury. Dysregulation of RAS may result in both glomerular and tubulointerstitial injury through direct effects of angiotensin II (Ang II) type 1 receptor. Interventions that inhibit the activity of the RAS are renoprotective and may slow or even stop the progression of nephropathies. ${ }^{[5]}$

Irbesartan blocks the vasoconstrictor and aldosterone-secreting effects of Ang II by selective blocking of Ang II receptors. Ang II is a principal presser agent of the RAS which encourages renal reabsorption of sodium and plays an important role in acute kidney injury. ${ }^{[6]}$ There are controversies about renal effect of RAS, Chida et al.'s study reported an important effect of irbesartan in the reduction of serum uric acid with potential antioxidant effect through reduction of oxidative stress in high-risk hypertensive patients ${ }^{[7]}$ While Hsu et al. illustrated that irbesartan and other Ang II blockers are inferior in nephroprotective effect compared with angiotensin-converting enzyme inhibitors. ${ }^{[8]}$

It has been shown that renoprotective effect of irbesartan is through the improvement of podocyte function and reduction of renal inflammation. As well, the renoprotective effect of Ang II blockers is mediated through reduction of renal tissue damage that induced by diabetes mellitus or nephrotoxic agents through the ruling of renal hemodynamic and down-regulation of cytokines-induced glomerular injury ${ }^{[9]}$ Moreover, Ang II blocker slows the development of diabetic nephropathy through modulation of intraglomerular pressure as intrarenal Ang II induces glomerular hyperfiltration and renal injury. ${ }^{[10]}$

Therefore, the rational of the present study depends on the suggestion that anti-inflammatory agents might play a potential role in the reduction of GIN since; GIN is partially mediated by activation of Ang II receptors. ${ }^{[11]}$

Consequently, the aim of the present study was to demonstrate the renoprotective effect of irbesartan on GIN in rats concerning the oxidative stress.

\section{Materials and Methods}

\section{Animals}

Thirty male Sprague-Dawley rats were used, which obtained from the National Center for Drug Control and Research, the rat's age ranged from 2 to 3 months, and their body weight (BW) ranged from 200 to $350 \mathrm{~g}(268.00 \pm 25.01)$. The animals were kept with suitable temperature $25 \mathrm{C}$ and artificial $12 / 12$ light-dark cycle with free access to normal chow pellets and water ad libitum. Humane care was according to the guide of care and use of laboratory animal.

\section{Drugs}

The drugs which were used in the present study included, gentamicin ampoule (Garamycin $80 \mathrm{mg}$ Schering-Plough, China) and irbesartan tablet (Approval $150 \mathrm{mg}$ tablet, Macleoods Pharma USA, Inc., Plainsboro, NJ 08536), were purchased from private pharmacy.

\section{Study design}

After the acclimatization period of 1 week, the weight of rats was taken, and the rats were randomly divided into three groups, $(n=10)$. The study protocol and method for induction of nephrotoxicity were according to Singh et al.'s method. ${ }^{[12]}$

1. Group I $(n=10)$ : Rats treated with distilled water $(5 \mathrm{~mL} / \mathrm{kg}$ ) orally for 12 days and on day $6-12$ they received intraperitoneal (i.p.) injection of normal saline daily $(5 \mathrm{~mL} / \mathrm{kg})$

2. Group II $(n=10)$ : Rats treated with distilled water $(5 \mathrm{ml} / \mathrm{kg})$ orally for 12 days and on day $6-12$, they received intraperitoneal injection of gentamicin $100 \mathrm{mg} / \mathrm{kg}$

3. Group III $(n=10)$ : Rats treated with irbesartan $(10 \mathrm{mg} / \mathrm{kg})$ for 12 days and on day $6-12$ they received intraperitoneal injection of gentamicin $100 \mathrm{mg} / \mathrm{kg}$.

\section{Anthropometric variables}

The length of the rat was measured by graduated tape measure from nose to the anus in centimeter. Rat BW was measured by the specific digital balance in gram. Body mass index (BMI) is equal to the BW in grams over the square of length in $\mathrm{cm}, \mathrm{BMI}=\mathrm{BW}(\mathrm{g}) /$ length $(\mathrm{cm})^{2} \cdot{ }^{[13]}$

Estimated glomerular filtration rate (eGFR) was measured according to Schwartz formula, eGFR $=\mathrm{k} \times$ height $(\mathrm{cm}) /$ serum creatinine $(\mathrm{mg} / \mathrm{dl}), K=0.55 .{ }^{[14]}$

\section{Sample collection}

On the $11^{\text {th }}$ day, rat decapitation was done under anesthesia; the blood samples were kept in the gel tubes which centrifuged at $5000 / \mathrm{rpm}$ for $10 \mathrm{~min}$. The formed sera were kept at $-20^{\circ} \mathrm{C}$ to be assessed later. The kidney was separated and stored in normal saline solution. The isolated kidneys were fixed in $10 \%$ formalin buffer to preserve the tissue structure according to the paraffin methods. ${ }^{[15]}$ Scoring system of renal histopathological changes was done according to a previous experimental study. ${ }^{[16]}$

\section{Biochemical variables}

Blood urea and serum creatinine were estimated using specific kits (colorimetric assay kit, E-BC-K183, Elabsciences, USA) and (colorimetric assay kit, E-BC-k186, Elabsciences, USA), respectively, which 
expressed as $\mathrm{mg} / \mathrm{dL}$ ). Serum malondialdehyde (MDA), superoxide dismutase (SOD), glutathione reductase (GSH), neutrophil gelatinase-associated lipocalin (NGAL), kidney injury molecules (KIM-1), and cystatin-c were measured by ELISA kit methods according to the instruction of the manufacturer (Myo-bio source, USA).

\section{Statistical analysis}

Statistical Package for the Social Sciences Software (IBM SPSS Statistics for Windows version 20.0, 2014, IBM Corp., Armonk, NY, New York, USA,) was used for data analysis. Data of the present study were presented mean \pm standard deviation, and the variables were tested using unpaired Student's $t$-test between control and treated groups. One-way ANOVA test with post hoc test was used to investigate the significance of differences among different groups. Pearson correlation was applied to detect the correlation of the study parameters. KruskalWallis test was used for recognizing the significance of differences concerning the histopathological scoring. The levels of significance were regarded when $P<0.05$.

\section{Results}

The characteristics of the present study demonstrated that 28 out of 30 Sprague-Dawley rats were used in the final analysis due to $6.67 \%$ death rate; other characteristics are presented in Table 1.

\section{Renal and oxidative stress biomarkers during} gentamicin-induced nephrotoxicity

During GIN, rat BW was increased compared with the control group $P=0.04$. The BMI was increased in the gentamicin group compared with the control

Table 1: Demographic characteristics of the present study

\begin{tabular}{l|c}
\hline The characteristics & Mean \pm SD, $n(\%)$, other \\
\hline Type of the study & $\begin{array}{c}\text { Experimental, animal } \\
\text { model study }\end{array}$ \\
$\begin{array}{l}\text { Animal used } \\
\text { Type }\end{array}$ & Male Sprague-Dawley rats \\
Number of rats & 30 \\
Age (months) & $2-3$ \\
Body weight $(\mathrm{g})$ & $268.00 \pm 25.01$ \\
Death rate $\%$ & $2(6.67)$ \\
Agents used & $10(33.33)$ \\
Normal saline $(9 \%)+$ distilled water & $8(26.66)$ \\
Gentamicin $100 \mathrm{mg} / \mathrm{kg}+$ normal & \\
saline $(9 \%)$ & $10(33.33)$ \\
Gentamicin $100 \mathrm{mg} / \mathrm{kg}+$ irbesartan 10 & \\
mg/kg & Kidney \\
Tissue used & Inflammatory and \\
Biomarkers & antioxidant biomarkers \\
\hline
\end{tabular}

$\mathrm{SD}=$ Standard deviation
$P=0.001$. Blood urea was increased in the gentamicin group compared with the control group $P=0.001$, whereas serum creatinine was increased compared with the control group $P=0.001$. The estimated GFR was reduced in gentamicin group compared with the control $P=0.001$. Concerning the oxidative stress, there was significant increase in the MDA serum levels in gentamicin group compared with the control group $P=0.001$ as well, SOD but not GSH sera levels were reduced in gentamicin group compared with control group $P=0.001$ and $P=0.49$ correspondingly. Besides, KIM-1 was increased in the gentamicin group compared with the control group $P=0.0001$. NGAL serum level was not increased significantly compared with the control group $P=0.003$ [Table 2].

Effect of irbesartan on renal and oxidative stress biomarkers during gentamicin-induced nephrotoxicity

Irbesartan reduced rat BW and BMI compared with gentamicin group $P=0.001$. As well, irbesartan reduced blood urea and serum creatinine compared with gentamicin group $P=0.001$. Irbesartan improved estimated GFR compared with gentamicin group $P=0.002$. On the other hand, irbesartan reduced MDA and increased SOD sera levels compared with gentamicin group $P=0.001$ with no significant effect on GSH serum levels $P=0.07$. Remarkably, irbesartan reduced KIM-1 and NGAL sera levels compared with gentamicin group, $P=0.0001$ and $P=0.003$, respectively [Table 2].

Regarding biomarker of glomerular injury, cystatin $C$ serum level was increased in GIN compared with the control group $(0.69 \pm 0.03 \mathrm{mg} / \mathrm{dL})$, but irbesartan administration led to reduction of cystatin $C$ serum level to $0.8 \pm 0.01 \mathrm{mg} / \mathrm{dL}$ compared with gentamicin group $P<0.01$ [Figure 1].

During GIN, high blood urea levels were positively correlated with serum creatinine, MDA, KIM-1, and cystatin $C, P<0.05$, but it negatively correlated with estimated GFR and SOD $P<0.05$. Indeed, reduction of blood urea level through irbesartan coadministration was significantly correlated with the reduction of serum creatinine, MDA, KIM-1, and Cystatin C as well as elevation of SOD serum levels and the improvement of estimated GFR [Table 3].

Effect of irbesartan on renal histopathological changes during gentamicin-induced nephrotoxicity Control group showed normal renal tissue, but in gentamicin group, there were significant histopathological changes including sloughing of renal tubular epithelium, dilatation of tubules with atrophy of epithelium, and formation of proteinaceous cast inside the tubules. Irbesartan led to significant amelioration 
Table 2: Effect of irbesartan on rat biomarkers in gentamicin-induced nephrotoxicity compared with control

\begin{tabular}{|c|c|c|c|c|c|c|c|}
\hline \multirow[t]{2}{*}{ Variables } & \multirow[t]{2}{*}{ Control $(n=10)$} & \multirow[t]{2}{*}{$\mathrm{G}+\mathrm{S}(n=8)$} & \multirow[t]{2}{*}{$\mathrm{G}+\mathrm{I}(n=10)$} & \multicolumn{3}{|c|}{ Post-hoc test } & \multirow[t]{2}{*}{ ANOVA } \\
\hline & & & & A & B & C & \\
\hline Weight (g) & $268.00 \pm 25.01$ & $298.37 \pm 25.02$ & $269.54 \pm 26.21$ & $0.04^{\pi}$ & NS & $0.04^{\pi}$ & $0.024^{\Uparrow}$ \\
\hline Height $(\mathrm{cm})$ & $21.50 \pm 0.83$ & $21.51 \pm 0.84$ & $21.51 \pm 0.81$ & NS & NS & NS & 0.99 \\
\hline $\mathrm{BMI}\left(\mathrm{g} / \mathrm{cm}^{2}\right)$ & $0.58 \pm 0.02$ & $0.64 \pm 0.04$ & $0.58 \pm 0.03$ & $0.001^{*}$ & NS & $0.001^{*}$ & $0.001^{*}$ \\
\hline Blood urea (mg/dL) & $41.83 \pm 6.46$ & $56.87 \pm 9.45$ & $42.56 \pm 7.89$ & $0.001^{*}$ & NS & $0.001^{*}$ & $0.0005^{*}$ \\
\hline Serum creatinine $(\mathrm{mg} / \mathrm{dL})$ & $0.70 \pm 0.14$ & $1.08 \pm 0.20$ & $0.86 \pm 0.22$ & $0.001^{*}$ & NS & 0.04 & $0.01^{\uparrow}$ \\
\hline E-GFR (ml/min/1.73) & $16.89 \pm 4.21$ & $10.95 \pm 2.16$ & $13.75 \pm 2.45$ & $0.002^{*}$ & NS & NS & $0.003^{*}$ \\
\hline MDA (ng/mL) & $289.85 \pm 14.18$ & $408.11 \pm 22.8$ & $290.52 \pm 12.87$ & $0.001^{*}$ & NS & $0.001^{*}$ & $0.0001^{*}$ \\
\hline $\mathrm{SOD}(\mathrm{pg} / \mathrm{mL})$ & $48.12 \pm 7.92$ & $26.39 \pm 5.86$ & $39.98 \pm 6.39$ & $0.001^{*}$ & $0.04^{\pi}$ & $0.001^{*}$ & $0.0001^{*}$ \\
\hline $\mathrm{GSH}(\mu \mathrm{g} / \mathrm{mL})$ & $15.94 \pm 2.39$ & $13.89 \pm 2.94$ & $14.77 \pm 2.86$ & NS & NS & NS & NS \\
\hline $\mathrm{KIM}-1(p g / m L)$ & $73.78 \pm 6.29$ & $154.98 \pm 16.38$ & $77.56 \pm 6.98$ & $0.001^{*}$ & NS & $0.001^{*}$ & $0.0001^{*}$ \\
\hline $\mathrm{NGAL}(\mathrm{pg} / \mathrm{mL})$ & $15.78 \pm 3.07$ & $23.04 \pm 5.88$ & $16.67 \pm 3.98$ & $0.006^{*}$ & NS & $0.01^{\Uparrow}$ & $0.003^{*}$ \\
\hline
\end{tabular}

Table 3: Correlation of blood urea with renal biomarkers in gentamicin-induced nephrotoxicity concerning the effect of irbesartan

\begin{tabular}{|c|c|c|c|c|c|c|}
\hline \multirow[t]{2}{*}{ Variables } & \multicolumn{2}{|c|}{ Control } & \multicolumn{2}{|c|}{$\mathbf{G}+\mathbf{S}$} & \multicolumn{2}{|c|}{$\mathbf{G}+\mathbf{I}$} \\
\hline & $r$ & $P$ & $r$ & $P$ & $r$ & $P$ \\
\hline Serum creatinine $(\mathrm{mg} / \mathrm{dL})$ & 0.62 & 0.33 & 0.99 & $0.01^{\pi}$ & 0.96 & $0.01^{\Uparrow}$ \\
\hline GFR (ml/min/1.73) & 0.33 & 0.50 & -0.91 & $0.04^{\pi}$ & 0.89 & $0.04^{\pi}$ \\
\hline MDA (ng/mL) & 0.57 & 0.23 & 0.99 & $0.008^{*}$ & 0.99 & $0.01^{\pi}$ \\
\hline $\mathrm{SOD}(\mathrm{pg} / \mathrm{mL})$ & 0.72 & 0.09 & -0.99 & $0.001^{*}$ & -0.98 & $0.02^{n}$ \\
\hline $\mathrm{GSH}(\mu \mathrm{g} / \mathrm{mL})$ & 0.45 & 0.43 & -0.89 & 0.09 & -0.77 & 0.08 \\
\hline $\mathrm{KIM}-1(\mathrm{pg} / \mathrm{mL})$ & 0.86 & 0.06 & 0.89 & $0.02^{\Uparrow}$ & 0.85 & $0.01^{\pi}$ \\
\hline Cys-c (mg/dL) & 0.66 & 0.15 & 0.98 & $0.015^{\natural}$ & 0.96 & $0.01^{\Uparrow 1}$ \\
\hline
\end{tabular}

${ }^{*} P<0.01, " P<0.05$, unpaired $t$-test. $r=$ Correlation level, $P=$ Level of significance, $\mathrm{G}+\mathrm{S}=$ Gentamicin+saline, $\mathrm{G}+\mathrm{I}=$ Gentamicin + irbesartan, $\mathrm{GFR}=\mathrm{Glomerular}$ filtration rate, $\mathrm{MDA}=$ Malondialdehyde, $\mathrm{SOD}=$ Superoxide dismutase, GSH=Glutathione reductase, KIM-1=Kidney injury molecule-1, Cys-C=Cystatin-C

of renal histological architecture; it reduced medullary congestion and tubular necrosis, from score 4 to score 2 compared with gentamicin group [Figure 2].

Gentamicin produced significant tubular injury $(3.6 \pm 0.4)$ compared with the control $(0.7 \pm 0.01)$ this damage was reduced when combined with irbesartan to $(2.5 \pm 0.6)$ [Figure 3].

\section{Discussion}

The present study demonstrated that GIN led to significant nephrotoxicity in rats through increasing of blood urea, serum creatinine, and reduction of estimated GFR. As well, GIN was associated with distant organ damage with subsequent body fluid accumulations that causing peripheral edema and raising of BMI as supported by a previous study. As well, induction of oxidative stress is the most important pathway of GIN leading to lipid peroxidation and tubular damages. ${ }^{[17,18]}$

Alternatively, RAS plays an imperative role in the development of acute renal injury. RAS agonist is

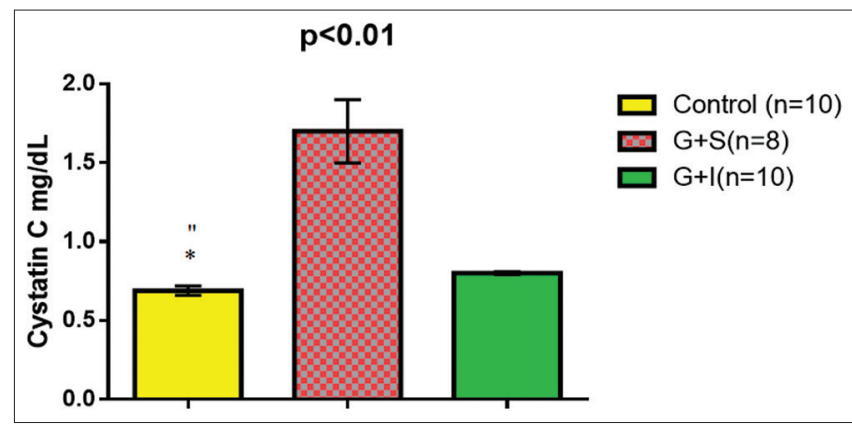

Figure 1: Irbesartan reduced cystatin $C$ serum levels during gentamicin-induced nephrotoxicity. ${ }^{*} P<0.01$ (control vs. gentamicin), ${ }^{* *} P>0.05$ (control vs. irbesartan)

regarded as a growth factor participates in hastening of renal damage due to activation of different inflammatory cytokines. Intrarenal Ang II is activated during toxic nephropathy and ischemic-reperfusion injury; therefore, Ang II antagonist has a renoprotective effect through the reduction of renal inflammation. ${ }^{[19]}$

These findings match with results of the present study that illustrated Ang II receptors blocker irbesartan leads to significant renoprotective effect through reduction of blood urea and improvement of estimated GFR since; irbesartan modulates Ang II bioactivity. ${ }^{[20]}$

Furthermore, the present study illustrated that irbesartan leads to the improvement of endogenous antioxidant capacity through elevation of GSH levels and reduction of MDA serum level. Recently, Maquigussa et al. confirmed the antioxidant effect of RAS blockers such as losartan which leads to significant renoprotective effect during ischemic renal injury. ${ }^{[21]}$

Alongside, irbesartan leads to important anti-inflammatory and renoprotective effect through reduction of NGAL and KIM-1 sera levels during GIN as illustrated in different studies. ${ }^{[9]}$ 


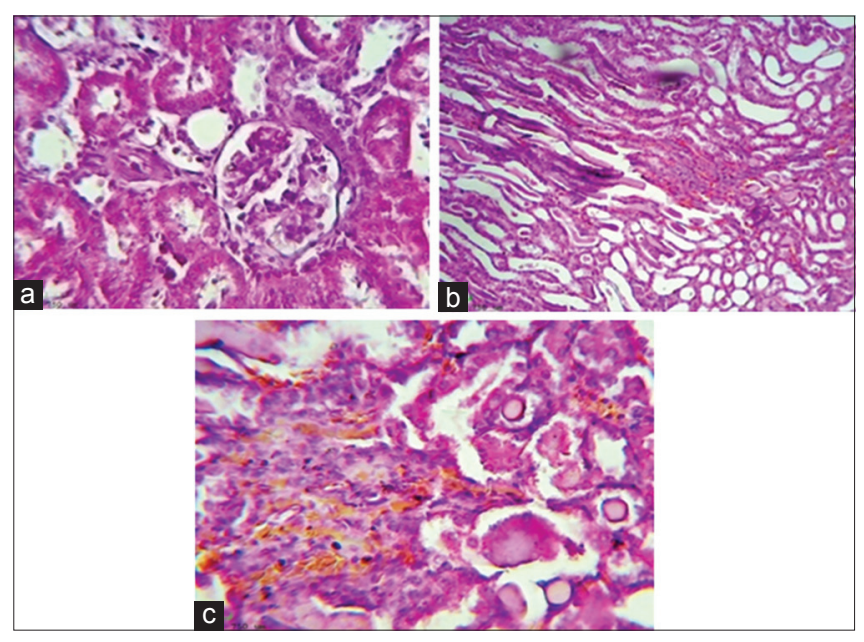

Figure 2: Irbesartan ameliorates renal histopathological changes during gentamicin-induced nephrotoxicity, (a) Histological picture shows normal looking renal tissue (Glomeruli and tubules, $\mathrm{H}$ and $\mathrm{E}, \times 400$ (reflect the effect of normal saline, (b) Histological section show severe medullary congestion (Score 4), detected on power of magnification $\times 100, \mathrm{H}$ and $\mathrm{E}$, which reflect the effect of gentamicin, (c) Histological section show moderate medullary congestion (Score 2 ), detected on power of magnification $\times 200, H$ and $E$, which reflect the effect of irbesartan

Likewise, the renoprotective effect of irbesartan is through reduction of renal tubular biomarkers (KIM-1 and NGAL) in patients with diabetic nephropathy. Beside, Valente et al. illustrated that losartan and other Ang-II receptor blockers attenuate acute kidney injury through inhibition of Ang II and interleukin-18 (IL-18) which linked with activation of renal oxidative stress. ${ }^{[22]}$ Unfortunately, Ang II and IL-18 sera levels were not evaluated in the present study.

Local RAS has been described to operate independently from their systemic counterpart. A local RAS, including all its components could have been shown in the proximal tubular cells of the kidney. Proximal tubular cells actively produce Ang II and also secrete angiotensinogen into the urine. Intraluminal angiotensinogen may be converted in the distal tubules to Ang II, and observations suggest that it leads to induction of sodium channels independent of aldosterone. Renal injury activates the local RAAS directly and indirectly. ${ }^{[23]}$

Entertainingly, clinical trials involving Irbesartan in hypertensive patients and in Diabetic Nephropathy Trial study illustrated that irbesartan revealed effective renoprotective effect through regulation of peroxisome proliferator-activator receptor-gamma (PPAR- $\gamma){ }^{[24]}$ Similarly, irbesartan shows a renoprotective effect independent on Ang II blocking effect which is most likely through PPAR- $\gamma$ since; blocking of PPAR- $\gamma$ abolish the renoprotective effect of irbesartan. In addition, irbesartan inhibits renal tumor growth factor- $\beta 1$ a mediator of renal damage through upregulation of PPAR- $\gamma$ which also improves renal antioxidant status. ${ }^{[25]}$

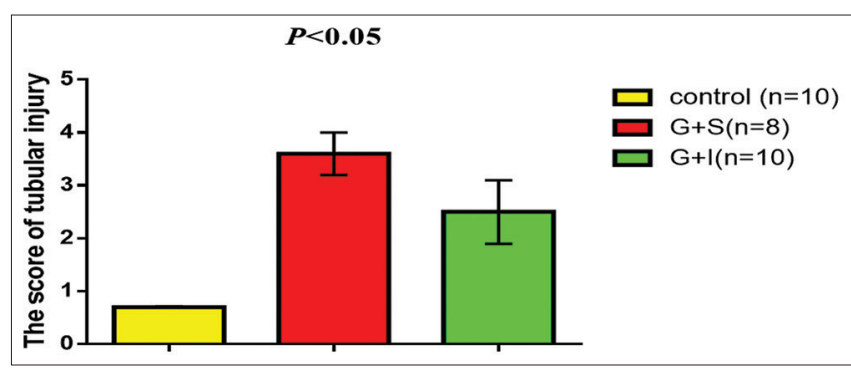

Figure 3: Irbesartan attenuates renal proximal tubular injury during gentamicin-induced nephrotoxicity

These findings may explain the renoprotective effect of irbesartan through reduction of renal injury biomarkers with minimal effect on lipid peroxidation biomarkers but; unfortunately, serum levels of PPAR- $\gamma$ were not assessed in the present study.

Therefore, the present study demonstrated that the antioxidant effect of irbesartan was the sole mechanism in attenuation of GIN. Nevertheless, Nasri study confirmed that therapy with reduced glutathione fails in prevention of GIN in spite of the reduction of lipid peroxidation thus; gentamicin produced dose-dependent effects in the progression of nephrotoxicity. Therefore, gentamicin induced-oxidative damage is not only the sole mechanism of GIN since; endoplasmic stress, membrane phospholipids and/or destabilization, activation of calcium-sensing receptors, and disturbance of cellular energy may be proposed mechanisms. ${ }^{[26]}$

Concerning the histopathological changes during GIN, gentamicin led to medullary congestion, interstitial infiltration, tubular epithelium sloughing, tubular necrosis, and glomerular damage compared with the control. These histopathological changes were correlated with the elevated KIM-1, NGAL, MDA, and reduced SOD, GSH sera levels as confirmed by different studies. ${ }^{[27]}$

Coadministration of irbesartan with gentamicin led to a significant reduction of GIN since; irbesartan leads to significant renoprotective effect through reduction of oxidative stress and augmentation of antioxidant activity. ${ }^{[28]}$

\section{Conclusion}

Irbesartan has renoprotective effect in attenuation of acute nephrotoxicity through modulation of oxidative stress and antioxidant capacity in rats.

\section{Acknowledgment}

The authors would like to thank teaching staff in the Department of Clinical Pharmacology for their cooperation and supports. 


\section{Financial support and sponsorship}

Nil.

\section{Conflicts of interest}

There are no conflicts of interest.

\section{References}

1. Al-Naimi MS, Rasheed HA, Hussien NR, Al-Kuraishy HM, Al-Gareeb AI. Nephrotoxicity: Role and significance of renal biomarkers in the early detection of acute renal injury. Journal of Advanced Pharmaceutical Technology \& Research 2019;10:95-9.

2. Cuzzocrea S, Mazzon E, Dugo L, Serraino I, Di Paola R, Britti D, et al. A role for superoxide in gentamicin-mediated nephropathy in rats. Eur J Pharmacol 2002;450:67-76.

3. Al-Kuraishy HM, Al-Gareeb AL, Rasheed HA. Antioxidant and anti-inflammatory effects of curcumin contribute into attenuation of acute gentamicin-induced nephrotoxicity in rats. Asian J Pharm Clin Res 2019;12:466-8.

4. Talebi A, Karimi A, Ouguerram K, Vahidi-Ataabadi N, Eshraghi-Jazi F, Mansouri A, et al. Lack of nephroprotective efficacy of Althaea officinalis flower extract against gentamicin renal toxicity in male rats. Int J Prev Med 2014;5:1360-3.

5. Heeba GH. Angiotensin II receptor blocker, losartan, ameliorates gentamicin-induced oxidative stress and nephrotoxicity in rats. Pharmacology 2011;87:232-40.

6. Mai HN, Chung YH, Shin EJ, Kim DJ, Jeong JH, Nguyen TT, et al. Genetic depletion of glutathione peroxidase-1 potentiates nephrotoxicity induced by multiple doses of cocaine via activation of angiotensin II AT1 receptor. Free Radic Res 2016;50:467-83.

7. Chida R, Hisauchi I, Toyoda S, Kikuchi M, Komatsu T, Hori Y, et al. Impact of irbesartan, an angiotensin receptor blocker, on uric acid level and oxidative stress in high-risk hypertension patients. Hypertens Res 2015;38:765-9.

8. Hsu FY, Lin FJ, Ou HT, Huang SH, Wang CC. Renoprotective effect of angiotensin-converting enzyme inhibitors and angiotensin II receptor blockers in diabetic patients with proteinuria. Kidney Blood Press Res 2017;42:358-68.

9. Hartner A, Cordasic N, Klanke B, Menendez-Castro C, Veelken R, Schmieder RE, et al. Renal protection by low dose irbesartan in diabetic nephropathy is paralleled by a reduction of inflammation, not of endoplasmic reticulum stress. Biochim Biophys Acta 2014;1842:558-65.

10. Sasso FC, Carbonara O, Persico M, Iafusco D, Salvatore T, $\mathrm{D}^{\prime}$ Ambrosio $\mathrm{R}$, et al. Irbesartan reduces the albumin excretion rate in microalbuminuric type 2 diabetic patients independently of hypertension: A randomized double-blind placebo-controlled crossover study. Diabetes Care 2002;25:1909-13.

11. Funakoshi $Y$, Ichiki $T$, Takeda $K$, Tokuno T, Iino N, Takeshita A. Critical role of cAMP-response element-binding protein for angiotensin II-induced hypertrophy of vascular smooth muscle cells. J Biol Chem 2002;277:18710-7.

12. Singh AP, Junemann A, Muthuraman A, Jaggi AS, Singh N, Grover $\mathrm{K}$, et al. Animal models of acute renal failure. Pharmacol Rep 2012;64:31-44.

13. Al-Kuraishy HM, Al-Gareeb AI. Effects of rosuvastatin on metabolic profile: Versatility of dose-dependent effect. Journal of advanced pharmaceutical technology \& research 2019;10:33-41.

14. Gacka E, Życzkowski M, Bogacki R, Paradysz A, Hyla-Klekot L. The usefulness of determining neutrophil gelatinase-associated lipocalin concentration excreted in the urine in the evaluation of cyclosporine a nephrotoxicity in children with nephrotic syndrome. Dis Markers 2016;2016:6872149.

15. Thompson SM, Craven RA, Nirmalan NJ, Harnden P, Selby PJ, Banks RE. Impact of pre-analytical factors on the proteomic analysis of formalin-fixed paraffin-embedded tissue. Proteomics Clin Appl 2013;7:241-51.

16. Toprak O, Cirit M, Tanrisev M, Yazici C, Canoz O, Sipahioglu M, et al. Preventive effect of nebivolol on contrast-induced nephropathy in rats. Nephrol Dial Transplant 2008;23:853-9.

17. Al-Kuraishy HM, Al-Gareeb AI, Al-Naimi MS. Pomegranate protects renal proximal tubules during gentamicin induced-nephrotoxicity in rats. J Contemp Med Sci 2019;5:35-40.

18. Al-Kuraishy HM, Al-Gareeb AL, Al-Naimi MS. Pomegranate attenuates acute gentamicin-induced nephrotoxicity in sprague-dawley rats. Asian J Pharm Clin Res 2019;12:1-3.

19. Zhao S, Ghosh A, Lo CS, Chenier I, Scholey JW, Filep JG. Nrf2 deficiency upregulates intrarenal angiotensin-converting enzyme-2 and angiotensin 1-7 receptor expression and attenuates hypertension and nephropathy in diabetic mice. Endocrinology 2018;159:836-52.

20. Ghafil FA, Al-Zubaidi FA, Almedeny SA. Assessment of nephroprotective role of irbesartan against gentamicin induced nephrotoxicity in rats. Kufa J Vet Med Sci 2012;3:54-60.

21. Maquigussa E, Paterno JC, de Oliveira Pokorny GH, da Silva Perez M, Varela VA, da Silva Novaes A, et al. Klotho and PPAR gamma activation mediate the renoprotective effect of losartan in the 5/6 nephrectomy model. Front Physiol 2018;9:1033.

22. Valente AJ, Yoshida T, Murthy SN, Sakamuri SS, Katsuyama M, Clark RA, et al. Angiotensin II enhances AT1-nox 1 binding and stimulates arterial smooth muscle cell migration and proliferation through AT1, nox 1, and interleukin-18. Am J Physiol Heart Circ Physiol 2012;303:H282-96.

23. Beutler KT, Masilamani S, Turban S, Nielsen J, Brooks HL, Ageloff $\mathrm{S}$, et al. Long-term regulation of $\mathrm{ENaC}$ expression in kidney by angiotensin II. Hypertension 2003;41:1143-50.

24. Parving $\mathrm{HH}$, Lehnert $\mathrm{H}$, Bröchner-Mortensen J, Gomis R, Andersen S, Arner P, et al. The effect of irbesartan on the development of diabetic nephropathy in patients with type 2 diabetes. N Engl J Med 2001;345:870-8.

25. Nakamura T, Mizuno S. The discovery of hepatocyte growth factor (HGF) and its significance for cell biology, life sciences and clinical medicine. Proc Jpn Acad Ser B Phys Biol Sci 2010;86:588-610.

26. Nasri H. Antioxidant therapy to ameliorate chronic kidney disease induced by oxidative stress; An updated mini-review. J Prev Epidemiol 2017;2:1-5.

27. Al-Kuraishy HM, Al-Gareeb AI, Hussien NR. Synergistic effect of berberine and pentoxifylline in attenuation of acute kidney injury. International Journal of Critical Illness and Injury Science 2019;9:69-76.

28. Chander V, Singh D, Tirkey N, Chander H, Chopra K. Amelioration of cyclosporine nephrotoxicity by irbesartan, Aselective AT1 receptor antagonist. Ren Fail 2004;26:467-77. 